ILL-(TH)-99-02

hep-th/9904104

\title{
Superstring Perturbation Theory and Ramond-Ramond Backgrounds
}

\author{
David Berenstein* \\ and \\ Robert G. Leigh ${ }^{\dagger}$ \\ Department of Physics \\ University of Illinois at Urbana-Champaign \\ Urbana, IL 61801
}

September 6, 2018

\begin{abstract}
We consider perturbative Type II superstring theory in the covariant NSR formalism in the presence of NSNS and RR backgrounds. A concrete example that we have in mind is the geometry of D3-branes which in the near-horizon region is $A d S_{5} \times S_{5}$, although our methods may be applied to other backgrounds as well. We show how conformal invariance of the string path integral is maintained order by order in the number of holes. This procedure makes uses of the FischlerSusskind mechanism to build up the background geometry. A simple formal expression is given for a $\sigma$-model Lagrangian. This suggests a perturbative expansion in $1 / g^{2} N$ and $1 / N$. As applications, we consider at leading order the mixing of RR and NSNS states, and the realization of the spacetime supersymmetry algebra.
\end{abstract}

*email:berenste@hepux0.hep.uiuc .edu

$\dagger$ email:rgleigh@uiuc.edu 


\section{Introduction}

The quantization of superstrings in non-trivial backgrounds has become an urgent problem since it has been realized that there is a direct relationship to the strong coupling dynamics of gauge field theories. In particular, one is often interested in conformal field theories which are related to spacetimes which contain an anti-deSitter factor.[1] For examples with $A d S_{3}$ and backgrounds of NSNS fields, several cases have been worked out [2, 3, 4, 5, 6] for finite $N$. The more interesting case with RR backgrounds, corresponding to configurations of D-branes, are much more difficult. Work on $A d S_{3}$ has been presented in Ref. [7], while $A d S_{5} \times S^{5}$ has been discussed in the GreenSchwarz formalism 8, 9]. Ref. [10] discusses the latter in the context of an expansion around flat spacetime.

In this paper, we consider RR backgrounds in the covariant RNS formalism. We begin with the theory in flat spacetime and demonstrate, that in the large $N$ limit, string perturbation theory organizes itself into a $\sigma$-model which is exactly conformal. This procedure may be thought of as summing of worldsheet holes, with closed string loops suppressed because of large $N$. We check conformal invariance of the string path integral explicitly at leading non-trivial order, an application of the Fischler-Susskind mechanism. [11] The $\sigma$-model is of a non-standard type because of the RR background, but can be written formally in a way which is useful for perturbative calculations.

The case of $A d S_{5} \times S^{5}$ is of particular interest. This model has a great deal of symmetry and it is possible that a free conformal field theory description exists, perhaps similar to the NS backgrounds with $A d S_{3}$. There are a number of oddities, centering around the properties of superconformal ghosts, as well as the properties of the spacetime supersymmetry algebra, which remain elusive. 12 The present construction however is certainly not such an exact description, and must be discussed in the context of a weak curvature expansion. The analysis may be applied to other cases as well, such as the $D 1-D 5$ system, but we confine our attention here to $A d S_{5} \times S^{5}$.

The paper is organized as follows. In section 2, we discuss general properties of string perturbation theory and demonstrate that in the large $N$ limit, the leading effect corresponds to a sum over worldsheet boundaries. We argue that these effects can be accounted for by a formal $\sigma$-model which includes the $\mathrm{RR}$ background. In order to define the $\sigma$-model it is necessary to introduce an operator which acts like the square-root of the picture-changing operator, $P_{1 / 2}$. In section 3 , we discuss the conformal invariance of this $\sigma$-model at 
lowest order in $\alpha^{\prime}$ and demonstrate that the Fischler-Susskind mechanism properly resums the background geometry. In section 4, we discuss some simple aspects of the spectrum of excitations around this background, and demonstrate that at a given level, R and NS states mix. In section 5, we consider the realization of spacetime symmetry charges in terms of worldsheet currents and comment on the supersymmetry algebra. Our analysis here is limited by our incomplete knowledge of the properties of $P_{1 / 2}$. Further comments and speculations are reserved for the final section.

\section{Background of D3-branes: Summing over Boundaries}

The basic idea here for computing the partition function is familiar from field theory: consider a scalar field theory, with a background field turned on. The one-loop determinant may be thought of as being built up by summing over multiple insertions of the background.

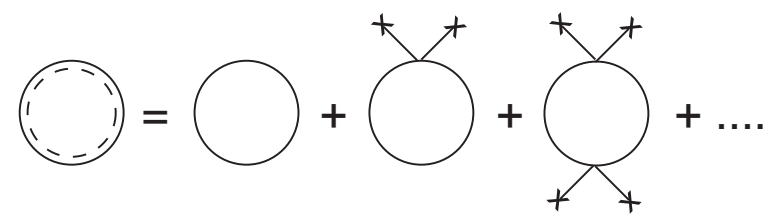

Indeed, in string theory in NSNS backgrounds, it is well known that summing over multiple insertions of vertex operators simply exponentiates those vertex operators, resulting in a $\sigma$-model. Here, we repeat this analysis in the case of interest, with RR-backgrounds, and we will discover in exactly what sense the RR vertex operator exponentiates. We will find that it is possible to write a formal expression (involving one term) that quantifies the effect of the RR-background. This is necessarily formal, because it involves an operator which changes ghost number in a non-standard way. The alternative is to remove this operator, but at the expense of introducing an infinite number of non-local operators into the " $\sigma$-model". The utility of the formal expression is that it automatically generates the correct combinatorics, and has a clear interpretation for any non-zero S-matrix element.

Let us begin by discussing the classical geometry exterior to a collection of $N$ D3-branes. The background is given by

$$
d s^{2}=f(r)^{1 / 2} d x_{\perp}^{2}+f(r)^{-1 / 2} d x_{\|}^{2}
$$


where $f(r)=1+R_{s}^{4} / r^{4}, r$ being the radial perpendicular coordinate. The radius of curvature satisfies $R_{s}^{4}=4 \pi g N \alpha^{\prime 2}$. In addition, there is a self-dual RR five-form fieldstrength, with total flux $N$, and the dilaton is constant. One notes that for large $g N$, the curvature of spacetime is everywhere small, and therefore solving the equations of motion at first order in $\alpha^{\prime}$ is a very good approximation. Furthermore, it has been argued from spacetime considerations that the near-horizon geometry is exact to all orders in $\alpha^{\prime}$ and $g$.

Our first task is to understand these results directly from string perturbation theory, where we integrate open string loops to all orders. In fact, the parameter that controls this perturbative expansion is $R_{s} / r$, so we will consider very large $r$ : we are expanding around flat spacetime. Furthermore, for $R_{s}^{2}>>\alpha^{\prime}$, it is possible to neglect the effects of higher string modes, even in the near-horizon limit.

In principle, since we are including open string loops to all orders, it would seem that we also need to include closed string loops. However, closed string loops are suppressed in large $N$ : they contribute at order $g^{2} \sim(g N)^{2} \times \frac{1}{N^{2}}$, and can be safely ignored. Open string loops contribute at order $g N$ since the worldsheet boundary can be placed at any of the $N$ D-branes.

To begin, let us consider the effects of a single boundary. Polchinski 13 computed the tadpoles of the graviton and RR 4-form potential in the presence of a D-brane to determine that a single D-brane carried one unit of RR-charge. The same calculation can be used to deduce that the gravitational field at a distance $r$ from a D3-brane has strength $\tilde{G}(X)=R_{s}^{4} / 2 r^{4}$. In this calculation, the information about polarizations is lost. This information may be recovered by a consideration of scattering of gravitons off of a D-brane. [14, 15] The result is that the polarization manifestly preserves $S O(6) \times S O(3,1)$,

$$
g_{\mu \nu}(X)=\frac{R_{S}^{4}}{2 r^{4}} t_{\mu \nu} \equiv \frac{R_{S}^{4}}{2 r^{4}}\left(\delta_{\mu \nu}^{\perp}-\delta_{\mu \nu}^{\|}\right)
$$

This is the leading term in an expansion of the supergravity metric (11) and we will see that it is consistent from the point of view of the string path integral as well.

If we are at large $r$, we can think of this as a gravitational fluctuation, 
with (on-shell) vertex operator in the $(0,0)$ picture円

$$
\begin{aligned}
\delta V_{g}= & -\frac{2}{\alpha^{\prime}} t_{\mu \nu}\left(-G(X) \partial X^{\mu} \bar{\partial} X^{\nu}+\frac{\alpha^{\prime}}{2}(\psi \cdot \partial G(X)) \psi^{\mu} \bar{\partial} X^{\nu}\right. \\
& \left.+\frac{\alpha^{\prime}}{2} \partial X^{\mu}(\tilde{\psi} \cdot \partial G(X)) \tilde{\psi}^{\nu}-\left(\frac{\alpha^{\prime}}{2}\right)^{2} \psi^{\lambda} \psi^{\mu} \tilde{\psi}^{\rho} \tilde{\psi}^{\nu} \partial_{\lambda} \partial_{\rho} G(X)\right)
\end{aligned}
$$

We will find that it is necessary to modify this vertex operator by contact terms; we will see that this is equivalent to going to $N=1$ superspace.

The $R R$ vertex operator in the $\left(-\frac{1}{2},-\frac{1}{2}\right)$-picture takes the form?

$$
\delta V_{R R}=\tilde{S}^{T} C \Gamma^{\mu_{1} \ldots \mu_{5}}\left(\frac{1+\Gamma_{-1}}{2}\right) S H_{\mu_{1} \ldots \mu_{5}} e^{-\phi / 2} e^{-\bar{\phi} / 2}
$$

where

$$
\Gamma^{\mu_{1} \ldots \mu_{5}} H_{\mu_{1} \ldots \mu_{5}}=\Gamma_{\|} \Gamma^{\alpha} \partial_{\alpha} G(X)
$$

The normalization of $V_{R R}$ may be determined by factorization.

The first step is to check under which conditions the operator (3) is welldefined; instead of defining it to be normal-ordered, we require that the singularities are absent. There are both quadratic divergences (contact terms) 16] and logarithmic divergences to consider. We will confine our attention to the $\partial X \bar{\partial} X$ part of the graviton vertex operator. The other terms follow from worldsheet supersymmetry.

The prefactor $G(X)$ will be dealt with by expansion around a fixed configuration: $G(X)=\langle G(X)\rangle+X^{\mu}\left\langle\partial_{\mu} G(X)\right\rangle+\ldots$ Point-splitting $\delta V_{g}$ (eq. (3)), we find

$$
\begin{aligned}
\int d^{2} z \delta V_{g}= & \int d^{2} z: \delta V_{g}:+\operatorname{tr} t 2 \pi \delta^{(2)}(\epsilon) \int d^{2} z: G(X): \\
& -\frac{1}{2} \ln |\epsilon|^{2} \int d^{2} z: \square G(X) t_{\mu \nu} \partial X^{\mu} \bar{\partial} X^{\nu}:+\ldots
\end{aligned}
$$

In the ellipsis are the fermion terms. There are higher order terms in $\alpha^{\prime}$, but they are all proportional to (derivatives of) $\square G$. Now, because $G(X)$ is a

\footnotetext{
${ }^{1}$ Our normalizations are $\left\langle X(z) X\left(z^{\prime}\right)\right\rangle=-\frac{\alpha^{\prime}}{2} \ln \left|z-z^{\prime}\right|^{2}$ and $\left\langle\psi(z) \psi\left(z^{\prime}\right)\right\rangle=1 /\left(z-z^{\prime}\right)$. Furthermore, $G(X)=\frac{\tilde{G}(X)}{4 \pi}$ (the $4 \pi$ is introduced to account for the differing normalizations of metric and vertex operator.)

${ }^{2} C$ is charge conjugation, $\Gamma_{-1}$ is 10 -dimensional chirality, and $\Gamma_{\perp(\|)}$ is chirality in the 6(4)-dimensional subspace.
} 
harmonic function, $\square G(X)$ vanishes, at least away from the branes. Thus $\delta V_{g}$ is defined up to a contact term. This contact term may be removed by modifying $\delta V_{g}$ by terms which vanish on the (tree-level) equations of motion. In fact, these modifications are equivalent to introducing $N=1$ superspace

$$
\mathbf{X}=X+i \theta \psi+i \bar{\theta} \tilde{\psi}+\theta \bar{\theta} F
$$

whereupon the vertex operator is written:

$$
\delta V_{g}=t_{\mu \nu} \int d^{2} z \int d^{2} \theta G(\mathbf{X}) D \mathbf{X}^{\mu} \bar{D} \mathbf{X}^{\nu}
$$

The new terms in the vertex operator which are relevant for the contact terms are

$$
G t_{\mu \nu}\left(\psi^{\mu} \bar{\partial} \psi^{\nu}+\tilde{\psi}^{\nu} \partial \tilde{\psi}^{\mu}+F^{\mu} F^{\nu}\right)
$$

The fermionic terms in the vertex operator are just the appropriate covariantizations

$$
\psi^{\mu}\left(G \bar{\partial} \delta_{\lambda}^{\nu}+\bar{\partial} X^{\nu} \partial_{\lambda} G\right) \psi^{\lambda},
$$

etc. These give additional contractions which cancel the contact term.

Thus we have shown that the vertex $\delta V_{g}$ is well-defined. The RR vertex operator requires no such treatment, as there are no contact terms; ; it is regular, as long as $\square G=0$. With these operators in hand, we are prepared to consider string worldsheets with multiple insertions. We will find that there are additional logarithmic short-distance singularities, which can be canceled by the Fischler-Susskind mechanism, namely by modifying the function $G$. This calculation appears in Section 3.

Let us consider the scattering of string states off of the D-branes at low momentum transfer. The momentum transfer is the Fourier transform of the coordinate $r$. We want to show that the contributions to this scattering are dominated by the exchange of massless states. The boundary on the D-brane may then be replaced in this factorization limit, by an insertion of a massless background vertex.

Indeed, this is what we would expect from looking at a Born-Oppenheimer approximation to the scattering. In essence, if each boundary has this property, then the relevant limit for calculating amplitudes involves long thin tubes attached to the brane, so each boundary is effectively shrunk to zero size, and can be replaced by a local insertion of a massless vertex operator,

\footnotetext{
${ }^{3}$ This assumes the spin field $S^{\alpha}$ is normal-ordered.
} 
which depends on $r$. This dependence comes about as each long thin tube extends from where the interaction takes place to the D-brane, and hence involves the Green's function $\sim \frac{1}{r^{4}}$ for the massless state in consideration.

The fact that the amplitude factorizes into a long thin tube for each boundary is a non-trivial statement, as one might imagine that there might be hard momentum flowing from one hole to another, thus making the boundaries big. We will argue that these effects cancel each other by supersymmetry. Indeed, in a low momentum scattering, the amplitude of external states factorizes onto a one point function of a vertex operator that is almost on-shell and massless, on a Riemann surface with $m>1$ holes. As we have more than one hole in the surface, the diagram without insertion is a self energy diagram of the brane configuration, and by supersymmetry vanishes; addition of the massless state insertion to this diagram is zero on-shell, as it probes the self-energy of the brane externally. The argument breaks down for $m=1$, as this probes the 'tree' level contribution to the mass of the brane, and this is the D-brane tension. Thus, the relevant limit of moduli space that contributes to the amplitude involves long thin tubes for all the insertions, and hence we get only effects from massless vertex operators in the full calculation.

Summing over this restricted moduli space of zero size holes integrates these vertex operator insertions over a Riemann surface with fixed complex structure. We sum over all inequivalent surfaces, and since the boundaries are all identical, we should divide out by the permutation symmetry to avoid overcounting. The sum over boundaries generates the series

$$
1+\sum_{m=1}^{\infty} \frac{1}{m !}\left(\int_{\Sigma} V_{B}\right)^{m}=\exp \int_{\Sigma} d^{2} z V_{B}
$$

with $V_{B}$ a massless string state generated by the boundary. That is, the sum over boundaries generates an expression which has a sigma model interpretation where we modify the action by

$$
S \rightarrow S+\int_{\Sigma} d^{2} z V_{B}(r)
$$

where $r$ is the impact parameter quantum field on the worldsheet. The sum over spin structures implies that $V_{B}$ is given by the combined graviton and self dual RR tadpole which we extracted in eqs. (8),(田) above.

From the point of view of string perturbation theory around flat spacetime, this corresponds to a partial resummation. As far as the metric is 
concerned, the exponentiation is standard. For the RR background, as discussed, the combinatorics are right for exponentiation, but the superconformal ghost factors make this problematic. A $\sigma$-model for these backgrounds may be written, formally, in several ways. The basic issue is that we wish to write an expression with zero ghost charge. In an S-matrix element, it would be convenient to take the external states in a fixed picture, and the insertion of eq. (12) implies that each term in $V_{B}$ should be in the same picture.

There may exist a field redefinition which mixes ghosts and matter fields similar to Ref. [17, 7] that would sidestep this problem but instead we propose to formally write the $\sigma$-model as

$$
S_{R R} \sim \int P_{1 / 2} \bar{P}_{1 / 2} V_{(-1 / 2,-1 / 2)}
$$

where $P_{1 / 2}\left(\bar{P}_{1 / 2}\right)$ increases the $\mathrm{L}(\mathrm{R})$-ghost charge by $1 / 2$ and satisfies $P_{1 / 2}^{2}=$ $P_{+1}\left(\bar{P}_{1 / 2}^{2}=\bar{P}_{+1}\right)$. Such operators are clearly not well-defined. However, this expression has two important properties: it generates the correct combinatorics, and is unambiguous for any non-zero S-matrix element. The basic point is that in an expansion of this exponential, the RR background vertex only contributes in pairs (on any topology). Using this $\sigma$-model, one can systematically compute perturbative corrections. For a given on-shell Smatrix element on a given topology, one introduces the correct background ghost charge explicitly, then takes each vertex operator, and each background vertex in the $(0,0)$ picture, defined as above. Further issues involving the properties of $P_{1 / 2}$ may be found in Sections 5,6.

An alternative procedure would be the following. Since the one-point function of $V_{R R}$ is zero on any topology, one could attempt to write non-local expressions of the form

$$
S_{R R} \sim \int V_{(+1 / 2,+1 / 2)} \int V_{(-1 / 2,-1 / 2)}+\ldots
$$

but the combinatorics of such an action are not correct: one needs to correct this at order $V^{4}$ and so on. An action with an infinite number of non-local terms is certainly not a terribly convenient representation.

\section{$3 \quad$ Fischler-Susskind and the $\sigma$-model}

Having discussed the general form of string perturbation theory in this background, we now give an explicit calculation of the modifications to the background which follow from conformal invariance of the string path integral. 
The calculation is an application of the Fischler-Susskind mechanism for a pair of colliding worldsheet holes. Thus we consider two background vertex operators, defined above, and bring them close together.

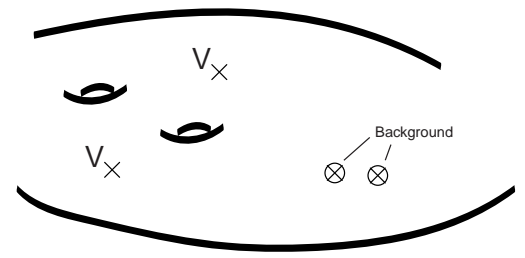

The calculation of the short-distance singularity is somewhat involved but straightforward, and we present here only the result. The quadratic divergences (contact terms) cancel exactly, given the modified form of the vertices discussed above. For simplicity, we write here the contributions to only the $\partial X \bar{\partial} X$ part

$$
\begin{array}{r}
\int d^{2} z \delta V_{g}(z) \cdot \int d^{2} z^{\prime} \delta V_{g}\left(z^{\prime}\right)= \\
-4 \pi \ln |\epsilon|^{2} \int d^{2} z\left(\partial X^{\mu} \partial_{\mu} G \bar{\partial} X^{\nu}\left(t^{2}\right)_{\nu}{ }^{\lambda} \partial_{\lambda} G\right. \\
+\bar{\partial} X^{\mu} \partial_{\mu} G \partial X^{\nu}\left(t^{2}\right)_{\nu}{ }^{\lambda} \partial_{\lambda} G \\
-\frac{1}{2}\left(\operatorname{tr} t^{2}\right) \partial X^{\mu} \partial_{\mu} G \bar{\partial} X^{\nu} \partial_{\nu} G-\partial X^{\mu} t_{\mu}^{\rho} \partial_{\rho} G \bar{\partial} X^{\nu} t_{\nu}{ }^{\lambda} \partial_{\lambda} G \\
\left.+(\partial G \cdot \partial G)\left(t^{2}\right)_{\mu \nu} \partial X^{\mu} \bar{\partial} X^{\nu}+G \square G t_{\mu \nu} \partial X^{\mu} \bar{\partial} X^{\nu}\right)
\end{array}
$$

Since $G(X)$ depends only on $r$, using the form of $t_{\mu \nu}$ discussed earlier this reduces to

$$
\begin{array}{r}
\int d^{2} z \delta V_{g}(z) \cdot \int d^{2} z^{\prime} \delta V_{g}\left(z^{\prime}\right)= \\
4 \pi \ln |\epsilon|^{2} \int d^{2} z\left(4 \partial_{\mu} G \partial_{\nu} G-(\partial G \cdot \partial G) \eta_{\mu \nu}\right) \partial X^{\mu} \bar{\partial} X^{\nu}+\ldots
\end{array}
$$

which is in the form of a graviton vertex operator.

There is also a logarithmically divergent contribution of the same form from two background RR vertices. After some Dirac algebra, we find

$$
\begin{array}{r}
\int d^{2} z \delta V_{R R}(z) \cdot \int d^{2} z^{\prime} \delta V_{R R}\left(z^{\prime}\right)= \\
4 \pi \ln |\epsilon|^{2} \int d^{2} z\left(-4 \partial_{\mu} G \partial_{\nu} G+2(\partial G \cdot \partial G) t_{\mu \nu}\right) \partial X^{\mu} \bar{\partial} X^{\nu}
\end{array}
$$


To obtain this result, the normalization of the $\mathrm{RR}$ vertex operator is required, as given in (14), and discussed in Section 5 .

The total logarithmic divergence in the graviton channel is then of the form

$$
4 \pi \ln |\epsilon|^{2} \int d^{2} z(\partial G \cdot \partial G)\left(\delta_{\mu \nu}^{\perp}-3 \delta_{\mu \nu}^{\|}\right) \partial X^{\mu} \bar{\partial} X^{\nu}+\ldots
$$

Note that the terms in $\partial r \bar{\partial} r$ have cancelled precisely between NS and R contributions.' In this sense, we have started in the "right" coordinate system.

The divergence (18) may be removed by modifying the graviton background through the addition of a term

$$
-2 \pi\left(\frac{2}{\alpha^{\prime}}\right) G^{2}(X)\left(\delta_{\mu \nu}^{\perp}-3 \delta_{\mu \nu}^{\|}\right) \partial X^{\mu} \bar{\partial} X^{\nu}+\ldots
$$

where the ellipsis contains fermionic terms, determined by supersymmetry. These terms have a logarithmically divergent self-contraction which exactly cancels that of eq. (18). To see this, one can use eq. (6) with $G$ replaced by $-2 \pi G^{2}$ : since $G^{2}$ is not harmonic, there is a non-zero contribution from $\square G^{2}$.

At this order then, the graviton background has the form

$$
\begin{array}{r}
\frac{1}{2 \pi \alpha^{\prime}}\left\{\left(\tilde{G}-\frac{1}{2} \tilde{G}^{2}\right) \delta_{\mu \nu}^{\perp}+\left(-\tilde{G}+\frac{3}{2} \tilde{G}^{2}\right) \delta_{\mu \nu}^{\|}\right\} \partial X^{\mu} \bar{\partial} X^{\nu}+\ldots \\
\simeq \frac{1}{2 \pi \alpha^{\prime}}\left\{\left(f^{1 / 2}-1\right) \delta_{\mu \nu}^{\perp}+\left(f^{-1 / 2}-1\right) \delta_{\mu \nu}^{\|}\right\} \partial X^{\mu} \bar{\partial} X^{\nu}+\ldots
\end{array}
$$

where $f=1+2 \tilde{G}(r)$ is the harmonic function appearing in the supergravity metric. Thus we see that at lowest order, the Fischler-Susskind mechanism does build up properly the background geometry of the 3-branes. Similar calculations may be performed without difficulty to demonstrate the appropriate modification of the RR background. We believe that this works to all orders in $\frac{R_{s}}{r}$; comments in this regard may be found in a later section of this paper. Certainly, this is not in disagreement with spacetime arguments that the near-horizon geometry is exact. [18, 19]

We present the following worldsheet argument that this is exact to all orders in $R_{s} / r$. Since $\alpha^{\prime} / R_{s}^{2}$ is small, it is useful to consider an expansion of the $\sigma$-model in powers of curvature. In order to simplify this expansion, one goes to normal coordinates around a point. In terms of this expansion

\footnotetext{
${ }^{4}$ Even if this were not true, a field redefinition can remove such terms.
} 
in curvature, the Ramond background is a first order perturbation, and the curvature correction to the metric is second order. In contrast, for the coordinates we were using before, which are not normal coordinates, the first correction comes from the Christoffel symbols, which are set to zero locally on the normal coordinate system.

This can be done for the full geometry, but for simplicity, we specialize now to the near horizon region. For $A d S_{5} \times S^{5}$, expanding around zero in the normal coordinates we have

$$
d s^{2} \sim \eta_{\mu \nu} d x^{\mu} d x^{\nu}+A x_{A d S}^{2} d x_{A d S}^{2}-A x_{S_{5}}^{2} d x_{S_{5}}^{2}+\ldots
$$

where $A$ is determined by the curvature of the space. Also

$$
V_{R R}=P_{1 / 2} \bar{P}_{1 / 2} S^{\alpha} \tilde{S}^{\beta} h_{\alpha \beta}
$$

with $h_{\alpha \beta} \sim\left[C\left(\Gamma_{A d S}+\Gamma_{S_{5}}\right)\right]_{\alpha \beta}$. The $\Gamma$ matrices used are products of the five gamma matrices tangent to $A d S_{5}$ and $S_{5}$ respectively.

Again we consider the collision of two background vertices. The algebra is straightforward, and we proceed by cancelling the logarithmic corrections from the square of the Ramond background, and the curvature selfcontraction. This produces the beta function for the graviton, and it reads

$$
\left(R_{\mu \nu}+\left(H^{2}\right)_{\mu \nu}\right) \log \left(|\epsilon|^{2}\right)=0
$$

We have used the $P_{1 / 2}$ operators for the Ramond vertex. As we have two Ramond vertex operators and to leading order they are on-shell (they are constant), the factors of $P_{1 / 2}$ give us a picture-changing operator, and the result is unambiguous. We thus reproduce the supergravity equations of motion for the graviton. This computation generalizes to any weakly coupled string theory at small curvature in the presence of RR backgrounds and gives the supergravity equations of motion.

This computation is one-loop in the $\sigma$-model and thus is correct to leading order in $\alpha^{\prime}$. Again according to [18, 19], this result is exact for the nearhorizon geometry to all orders in $\alpha^{\prime}$.

\section{Spectrum: Mixing of RR and NSNS states}

We have suggested in the above discussions that the $\sigma$-model may be consistently defined, and it is exactly conformal. The next step would be to discuss the spectrum of excitations around the background. Since the $\sigma$-model 
is known and exactly conformal, in principle one could write equations for vertex operators which make them of dimension $(1,1)$. The best we can do at present is to compute vertex operators for these excitations perturbatively in the normal coordinate expansion, starting with flat spacetime operators.

Note that in the approximation in which we are working, the spacing of states is as shown in the accompanying figure. The gap between levels is of order $\sqrt{\alpha^{\prime}}$ while the spacing between $S^{5}$ harmonics is of order $1 / R_{s}$.

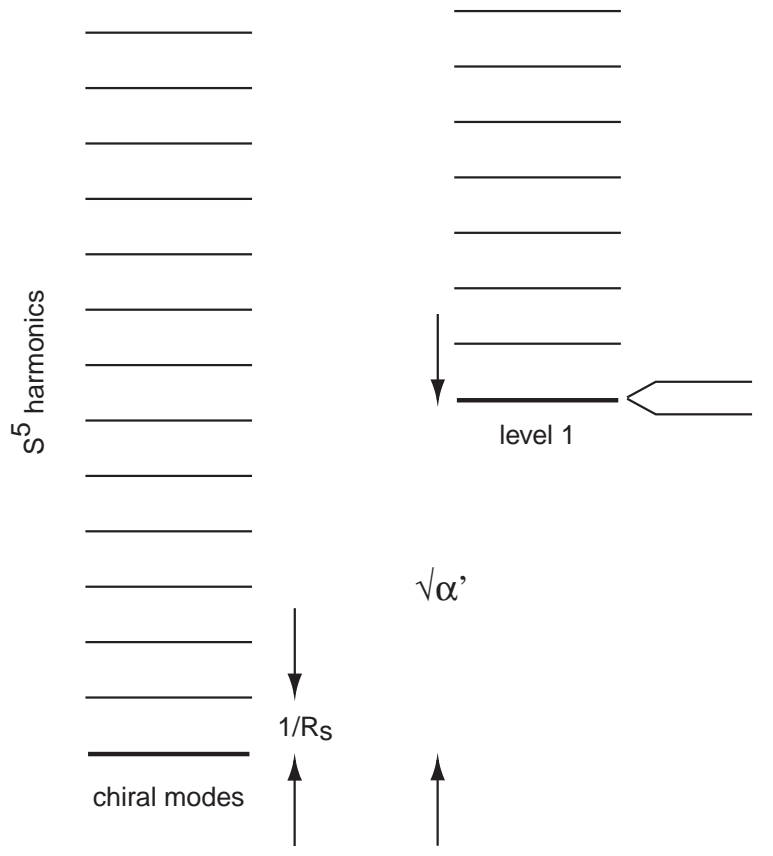

We expect this spectrum to be modified by the background. The leading effect is to mix states at the same level. Because of the RR background, the states which in flat space are RR and NSNS mix with one another, which means that there are no longer separately conserved number operators in the R- and NS-sectors. At most, we can expect that a linear combination is still a good quantum number, and thus organizes the spectrum, although it seems unlikely that even this is true at finite $N$.

Let us consider the one-loop partition function. In flat spacetime, bosonic symmetries which act on states come in the NS sector, and thus spacetime symmetries may be thought of as acting on, for example, the NSNS part of the partition function and the RR part separately. Once we turn on the background, this can no longer be true: there will be spacetime symmetries in the RR sector and thus at best we can classify states as bosonic or fermionic 
(which cancel by supersymmetry).

In this section, we simply demonstrate this mixing at lowest order in $\alpha^{\prime}$. The mixing is of order one. In principle, one can systematically compute higher order corrections. The basic idea is again an application of the Fischler-Susskind mechanism. We consider some arbitrary $(1,1)$ operator $\mathcal{O} e^{i p \cdot X}$ inserted on the worldsheet. We compute the effects of the background perturbatively by considering the effects of background vertex operators.

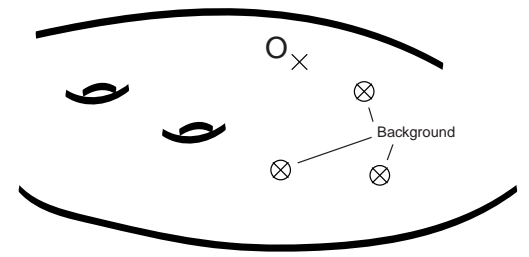

The leading effect will come from a single RR insertion

$$
\delta V_{R R}=\int d^{2} z P_{1 / 2} \bar{P}_{1 / 2}(C H)_{\alpha \beta} \tilde{S}^{\alpha} S^{\beta} e^{-\phi / 2} e^{-\bar{\phi} / 2}
$$

The operator product of $\delta V_{R R}$ with the vertex $\mathcal{O}$ will be of the form

$$
\int d^{2} z \frac{1}{\left|z-z^{\prime}\right|^{2}}(C H)_{\alpha \beta}\left[\bar{Q}^{\alpha} Q^{\beta} \mathcal{O} e^{i p \cdot X}\right]
$$

This is logarithmically divergent, and converts NS to R and vice versa. If need be this can be brought back to, say, the $(0,0)$ or $\left(-\frac{1}{2},-\frac{1}{2}\right)$-picture by applying the picture-changing operator.

Thus the leading order effect is to mix NS with R:

$$
\delta\left(\begin{array}{c}
\mathcal{O}_{N S} \\
\mathcal{O}_{R}
\end{array}\right)=\left(\begin{array}{cc}
0 & M \\
\tilde{M} & 0
\end{array}\right)\left(\begin{array}{c}
\mathcal{O}_{N S} \\
\mathcal{O}_{R}
\end{array}\right)
$$

where $M$ and $\tilde{M}$ are suitable (logarithmically divergent) mixings that may be computed from the operator products mentioned above (we consider an explicit example below.) In order to eliminate the divergence, we should modify the vertex operators, in two steps. First, we diagonalize

$$
\mathcal{O}_{D} \sim \alpha \mathcal{O}_{N S}+\beta \mathcal{O}_{R}
$$

\footnotetext{
${ }^{5}$ Note that at lowest order, we can assume that the picture changing operator is unchanged from its flat-space value. Higher order corrections must take this consistently into account.
} 
and then modify the operator $\mathcal{O}_{D}$ to eliminate the divergence. In the weak curvature limit, it is sufficient to consider

$$
: \mathcal{O}_{D} e^{i k \cdot X}: \rightarrow: \mathcal{O}_{D} e^{i k \cdot X}: e^{i \delta k \cdot X}
$$

Going beyond the weak curvature approximation is a difficult problem, equivalent to solving for the explicit form of the vertex operators in this background, and we do not attempt such an analysis here. The modification of the momenta accounts for the change in the mass of states in the background and is the only source of a logarithmic divergence within the vertex operator.

In principle, this analysis may be carried out for any state in the string spectrum, although it is technically challenging. We confine the discussion to a demonstration of the mixing effect for components of the massless gravity multiplet. For these states, the analysis reduces to that of Ref. 20] for supergravity in $A d S_{5} \times S_{5}$.

Begin then with massless states:

$$
\begin{aligned}
\mathcal{O}_{N S} & =\int d^{2} z h_{\mu \nu}^{(N S)}\left(-\partial X^{\mu}+i k \cdot \psi \psi^{\mu}\right)\left(-\bar{\partial} X^{\nu}+i k \cdot \tilde{\psi} \tilde{\psi}^{\nu}\right) e^{i k \cdot X} \\
\mathcal{O}_{R} & =\int d^{2} z h_{\alpha \beta}^{(R)} \tilde{S}^{\alpha} S^{\beta} e^{-\phi / 2} e^{-\bar{\phi} / 2} e^{i k \cdot X}
\end{aligned}
$$

We note that

$$
V_{B} \cdot \mathcal{O}_{N S}=-\int d^{2} z^{\prime} e^{-\phi / 2} e^{-\bar{\phi} / 2} e^{i k \cdot X} S^{\alpha} \tilde{S}^{\beta} \cdot 2 \pi \ln \epsilon h_{\mu \nu}^{(N S)} k_{\lambda} k_{\rho}\left(C \Gamma^{\lambda \nu} H \Gamma^{\rho \mu}\right)_{\alpha \beta}
$$

and

$$
V_{B} \cdot \mathcal{O}_{R}=\int d^{2} z 2 \pi \ln \epsilon h_{\alpha \beta}^{(R)}\left(C \Gamma^{\mu} H \Gamma^{\nu}\right)^{\alpha \beta} \psi^{\mu} \tilde{\psi}^{\nu} e^{-\phi} e^{-\bar{\phi}}
$$

These results are equivalent to a mixing matrix of the form:

$$
-2 \pi \ln \epsilon\left(\begin{array}{cc}
0 & h_{\mu \nu}^{(N S)} k_{\lambda} k_{\rho}\left(C \Gamma^{\lambda \nu} H \Gamma^{\rho \mu}\right)_{\alpha \beta} \\
-h_{\alpha \beta}^{(R)}\left(C \Gamma^{\mu} H \Gamma^{\nu}\right)^{\alpha \beta} & 0
\end{array}\right)
$$

and agrees with the supergravity analysis of [20]. This mixing suggests that the spacetime symmetries will not respect the splitting between R and NS.

\section{$5 \quad$ Spacetime Algebra}

Let us consider the realization of spacetime symmetries on the worldsheet and their algebra. In this discussion, there are some subtleties because of 
limited knowledge of $P_{1 / 2}$. However, modulo these subtleties, we are able to reproduce the correct spacetime supersymmetry in the curvature expansion around the near-horizon geometry. A fuller discussion will appear in a subsequent publication.

We know that in the presence of D-branes, half of the supersymmetry is broken, but is recovered in the near-horizon region as a superconformal symmetry. Our first task is to recover this fact in string perturbation theory.

It is natural to define supercharges

$$
\begin{aligned}
q^{\alpha} & =\oint d z S^{\alpha} e^{-\phi / 2} \\
\bar{q}^{\alpha} & =\oint d \bar{z} \tilde{S}^{\alpha} e^{-\bar{\phi} / 2}
\end{aligned}
$$

which certainly generate symmetries in flat spacetime. In a RR background, at lowest order in $\alpha^{\prime}$, there is only one linear combination

$$
Q^{\alpha} \sim P_{1 / 2} q^{\alpha}+\Gamma_{\|} \bar{P}_{1 / 2} \bar{q}^{\alpha}
$$

retained. To see this, we simply need to require that the supersymmetry annihilates the background

$$
Q^{\alpha} \cdot V_{B}=0
$$

This calculation in fact is another way to fix the normalization of the RR vertex operator. At lowest order, our lack of understanding of $P_{1 / 2}$ is irrelevant. At higher orders, we need to know $P_{1 / 2}$, and furthermore we expect that eq. (37) is further modified by functions of $r$. Once this happens, there is no possible splitting between holomorphic and anti-holomorphic fields. This effect can be computed from worldsheet considerations (given $P_{1 / 2}$ ) by a further application of Fischler-Susskind. From the spacetime point of view, they are determined by the requirement that $Q$ be associated to a Killing spinor.

We note the very important point here that the holomorphic and antiholomorphic charges mix with one another in the RR background. There is nothing really exotic about this: given a conserved current $J$, we can write $\bar{\partial} J+\partial \bar{J}=0$, and the spacetime charge $T=\oint d z J+\oint d \bar{z} \bar{J}$ is well-defined, being independent of contour. Only in special cases are $J$ and $\bar{J}$ separately conserved.

In the near-horizon limit, we expect that exactly this happens, and the other linear combination of spacetime supercharges $S^{\alpha}$, comes back. Indeed, in normal coordinates for the near-horizon case, we begin with 32 supercharges, and eq. (38) does not eliminate any. Instead, (38) defines the 
modifications to the supercharges in the presence of the background. We may write the Killing spinor equation in the form

$$
\nabla Q+Q \cdot V_{B}=0
$$

which is the more general form of (38). Consider solving this equation order by order in the curvature expansion:

$$
\begin{aligned}
Q^{\alpha} & =Q_{(0)}^{\alpha}+Q_{(1)}^{\alpha}+\ldots \\
\bar{Q}^{\alpha} & =\bar{Q}_{(0)}^{\alpha}+\bar{Q}_{(1)}^{\alpha}+\ldots
\end{aligned}
$$

The lowest order terms are the flat space expressions $Q_{(0)}=q, \bar{Q}_{(0)}=\bar{q}$. At next order, the RR background in $V_{B}$ gives a non-zero contribution to the second term in eq. (39) which is proportional to $\bar{P}_{1 / 2} \bar{q}^{\alpha}$. Since in the normal coordinate expansion, the Christoffel symbols vanish, (39) then implies that

$$
Q_{(1)} \sim \oint d \bar{z} \bar{P}_{1 / 2} X \cdot \Gamma \tilde{S}
$$

with a similar expression for $\bar{Q}_{(1)}$. Thus we see that indeed the supercharge has both holomorphic and antiholomorphic contributions. This is precisely what we need to reproduce the spacetime algebra. With the expressions that we have given, one may check the following commutators are induced by the worldsheet representation:

$$
\begin{aligned}
& \left\{Q^{\alpha}, \bar{Q}^{\beta}\right\} \sim \oint\left(\Gamma_{\mu \nu}\right)^{\alpha \beta}\left(X^{\mu} \cdot \partial X^{\nu}-X^{\mu} \cdot \bar{\partial} X^{\nu}\right)+\ldots \\
& \left\{Q^{\alpha}, Q^{\beta}\right\} \sim \oint\left(\Gamma_{\mu}\right)^{\alpha \beta} \partial X^{\mu}+\ldots
\end{aligned}
$$

In general, in order to compute such effects precisely, we need to understand in more detail the operator $P_{1 / 2}$. We believe that these obstacles can be overcome.

\section{Conclusions and discussion}

In this paper, we have analyzed the background produced by D3-branes. We have discussed how a $\sigma$-model description can be used. The background geometry is recovered by summing over boundaries of worldsheets, and systematically applying the Fischler-Susskind mechanism. Our results suggest 
to us that an exact string conformal field theory description may exist. In order to 'derive' such a description from perturbation theory requires further understanding of the square root of the picture changing operator. Our results indicate that this is not such an unnatural object. With this operator we can construct a perturbation expansion which is independent of the zero mode of the bosonized superconformal ghost system. This indicates that picture-changing is still a property of these $\sigma$-models, but the detailed form is modified by the background.

It is plausible that the approach of [7] is related to these remarks. The case of $A d S_{3}$ discussed there is considerably different however. In particular, there are half as many supersymmetries as for $A d S_{5}$, and consequently more freedom to choose pictures, and as well the target space of the $\sigma$-model is a group manifold. It seems difficult to imagine that a free-field realization exists in that the mixing of holomorphic and anti-holomorphic currents would seem to invalidate the idea of a spectrum generating algebra.

Acknowledgments: We wish to thank F. Larsen for collaboration at an early stage of this work. Research supported in part by the United States Department of Energy grant DE-FG02-91ER40677 and an Outstanding Junior Investigator Award.

\section{References}

[1] J. Maldacena, "The Large $N$ limit of superconformal field theories and supergravity," Adv. Theor. Math. Phys. 2 (1998) 231, hep-th/9711200.

[2] A. Giveon, D. Kutasov, and N. Seiberg, "Comments on string theory on $A d S_{3}, "$ Adv. Theor. Math. Phys. 2 (1998) 733-780, hep-th/9806194.

[3] S. Elitzur, O. Feinerman, A. Giveon, and D. Tsabar, "String theory on $A d S_{3} \times S^{3} \times S^{3} \times S^{1}, "$ hep-th/9811245.

[4] D. Kutasov, F. Larsen, and R. G. Leigh, "String theory in magnetic monopole backgrounds," hep-th/9812027.

[5] J. de Boer, H. Ooguri, H. Robins, and J. Tannenhauser, "String theory on $A d S_{3}, " J H E P 12$ (1998) 026, hep-th/9812046.

[6] D. Kutasov and N. Seiberg, "More comments on string theory on $A d S_{3}$," hep-th/9903219. 
[7] N. Berkovits, C. Vafa, and E. Witten, "Conformal field theory of AdS background with Ramond-Ramond flux," hep-th/9902098.

[8] R. R. Metsaev and A. A. Tseytlin, "Type IIB superstring action in Ad $S_{5} \times S^{5}$ background," Nucl. Phys. B533 (1998) 109, hep-th/9805028.

[9] R. Kallosh and J. Rahmfeld, "The GS string action on $A d S_{5} \times S^{5}$," Phys. Lett. B443 (1998) 143, hep-th/9808038.

[10] A. Rajaraman and M. Rozali, "On the quantization of the GS string on $A d S_{5} \times S^{5}$," hep-th/9902046.

[11] W. Fischler and L. Susskind, "Dilaton Tadpoles, String Condensates And Scale Invariance," Phys. Lett. B171 (1986) 383.

[12] D. Berenstein, R. G. Leigh, and F. Larsen, "unpublished,".

[13] J. Polchinski, "Dirichlet Branes and Ramond-Ramond charges," Phys. Rev. Lett. 75 (1995) 4724-4727, hep-th/9510017.

[14] I. R. Klebanov and L. Thorlacius, "The Size of p-branes," Phys. Lett. B371 (1996) 51-56, hep-th/9510200.

[15] S. S. Gubser, A. Hashimoto, I. R. Klebanov, and J. M. Maldacena, "Gravitational lensing by p-branes," Nucl. Phys. B472 (1996) 231-248, hep-th/9601057.

[16] M. B. Green and N. Seiberg, "Contact Interactions In Superstring Theory," Nucl. Phys. B299 (1988) 559.

[17] N. Berkovits, "Covariant quantization of the Green-Schwarz superstring in a Calabi-Yau background," Nucl. Phys. B431 (1994) 258-272, hep-th/9404162.

[18] T. Banks and M. B. Green, "Nonperturbative effects in $A d S_{5} \times S^{5}$ string theory and $d=4$ SUSY Yang-Mills," JHEP 05 (1998) 002, hep-th/9804170.

[19] R. Kallosh and A. Rajaraman, "Vacua of M theory and string theory," Phys. Rev. D58 (1998) 125003, hep-th/9805041. 
[20] H. J. Kim, L. J. Romans, and P. van Nieuwenhuizen, "The Mass Spectrum Of Chiral N=2 d =10 Supergravity on $S^{5}$," Phys. Rev. D32 (1985) 389. 